\title{
Evaluation and Validation of Utility of BD Glucose Vacutainer for Glycosylated Hemoglobin Assay for Timely Therapeutic Management of Diabetes Mellitus
}

B. Sheshu Kumar ${ }^{1}$, Sachin Dominic ${ }^{1}$, Siraj Ahmed Khan ${ }^{1}$, N. N. Sreedevi ${ }^{1}$, B. Yadagiri ${ }^{1}$, M. Vijaya Bhaskar ${ }^{1}$, K.S.S. Sai $\mathrm{Baba}^{1}$, Shaik Mohammad Naushad ${ }^{2}$ and Iyyapu Krishna Mohan ${ }^{3 *}$

${ }^{1}$ Department of Biochemistry, Nizam's Institute of Medical Sciences, Panjagutta, Hyderabad, India

${ }^{2}$ Department of Biochemical Genetics, Sandor Lifesciences Pvt Ltd, Banjara Hills, Hyderabad, India

${ }^{3}$ Associate Professor, Department of Biochemistry, Nizam's Institute of Medical Sciences, Panjagutta, Hyderabad, India

DOI: $10.36348 /$ sijb.2019.v02i12.006 $\quad$ | Received: 19.12.2019| Accepted: 26.12.2019| Published: 30.12 .2019

*Corresponding author: Dr. Iyyapu Krishna Mohan

\section{Abstract}

Blood glucose and glycosylated haemoglobin are the two vital biochemical tests widely prescribed for the therapeutic management of diabetes and require different anticoagulants during specimen collection. If both tests can be performed using single vacutainer, it reduces the laboratory costs at a larger scale and facilitates timely intervention. The anticoagulants $\mathrm{NaF} / \mathrm{Na}_{2}$ EDTA and K2 EDTA are used conventionally for blood glucose and HbA1c estimations respectively for specimen collection. In the current study we have investigated whether $\mathrm{NAF} / \mathrm{Na}_{2}$ EDTA can be used for $\mathrm{HbA1c}$ assay without the need of separate vacutainer i.e.K2EDTA. A total of 125 subjects (25 non-diabetic and 100 diabetic) were enrolled for this study. Parallel samples were collected in $\mathrm{NaF} / \mathrm{Na}_{2}$ EDTA and K2 EDTA to compare HbA1c levels in both the anticoagulants. The stability of HbA1c was elucidated in both anticoagulants till $72 \mathrm{hrs}$ of collection. The HbA1c levels in both anticoagulants showed excellent correlation $\left(\mathrm{R}^{2}\right.$ : 0.998). Bland and Altman analysis revealed that the bias between methods is -0.22 to $+0.22 \%$. The changes in HbA1c levels were unaltered till $72 \mathrm{hrs}$ of collection in $\mathrm{NaF} / \mathrm{Na}_{2}$ EDTA and K2EDTA (mean SD: 0.066 vs. 0.058; \%CV: 0.94 vs. 0.81). These results conclude that the same vacutainer used for glucose estimation can be used even for HbA1c till $72 \mathrm{hr}$ of collection thus preventing the need of second sampling specifically in monitoring diabetic control. This study emphasized that a single vacutainer of $\mathrm{NaF} / \mathrm{Na}_{2}$ EDTA can be used safely for both glucose and $\mathrm{HbA1c}$ assays.

Keywords: NaF/Na2 EDTA; K2 EDTA; Glucose vacutainer; HbA1c assay; Diabetes.

Copyright @ 2019: This is an open-access article distributed under the terms of the Creative Commons Attribution license which permits unrestricted use, distribution, and reproduction in any medium for non-commercial use (NonCommercial, or CC-BY-NC) provided the original author and source are credited.

\section{INTRODUCTION}

The normal adult haemoglobin $\mathrm{HbA}$ consists of $2 \alpha$ and $2 \beta$ chains $(\alpha 2 \& \beta 2$ ) and make upto $97 \%$ of normal adult haemoglobin. By the process of post translational modification, $\mathrm{HbA}$ is modified into minor types- HbAla, HbA1b, HbA1c, of which HbA1c is the abundant form. $\mathrm{Hb} \mathrm{A} 1 \mathrm{c}$ is formed by a non enzymatic glycation process and occurs in two steps i.e reversible binding as an aldimine/schiff base and irreversible binding through amadori rearrangement to form a ketoamine linkage [1]. The process of post-translational modification of $\mathrm{HbA}$ to $\mathrm{HbA} 1 \mathrm{c}$ occurs at a slow rate throughout the lifespan of RBC. In 1976, HbA1c assay is proposed for the first time as a biomarker for monitoring the levels of glucose among diabetic patients [2].
In recent years, globally India has emerged as one of the epicentres of diabetic mellitus pandemic with more than 69.2 million diabetic individuals in a total population of 1339.2 million accounting for an incidence of 5.2\% [3]. Glycosylated haemoglobin (HbA1c) and fasting blood glucose are the two standard biochemical tests that are used for the diagnosis and therapeutic management of diabetes. Physicians are tailoring the diabetes therapy based on HbA1c levels in type 2 diabetes with HbA1c levels between 7.0-7.4\% for monotherapy (metformin preferably), $\mathrm{HbA} 1 \mathrm{c}$ between $8.0-8.4 \%$ for dual therapy and $\mathrm{HbA} 1 \mathrm{c}$ between 9.0-9.4\% for triple therapy [4]. Poor glycemic control was reported to increase incidence of severe hypoglycaemia, diabetic ketoacidosis, and increase risk for microvascular complications such as nephropathy and neuropathy in type 1 diabetes [5]. Fasting blood glucose $>172 \mathrm{mg} / \mathrm{dl}$ and $\mathrm{HbA} 1 \mathrm{C}>9.0 \%$ were shown to 
be associated with microalbuminuria [6]. In view of the pivotal role of these two biochemical tests as surrogate markers for diabetic related complications, frequent monitoring is mandatory.

Sodium fluoride and potassium oxalate was the conventional anticoagulants for glucose vacutainer where in sodium fluoride acts as inhibitor of glycolytic enzymes. Recently, potassium oxalate is replaced by $\mathrm{Na}_{2}$ EDTA as it precipitates as calcium oxalate, which is toxic. A small pilot study on three diabetic and three non-diabetic samples evaluated the stability of HbA1c in EDTA, heparin, citrate and fluoride and found no significant changes when sample was stored at $-4^{\circ} \mathrm{C}$ for seven days [7]. Another study found no significant difference in HbA1c or its stability between parallel post-mortem samples collected in EDTA and sodium fluoride vacutainers and stored at $4^{\circ} \mathrm{C}$ [8].

As there are a very few studies that substantiate the use of glucose vacutainer for HbA1c and all the available studies have limitations such as sample size, lack of validation studies across broad coverage of HbA1c, and have not evaluated stability of $\mathrm{HbA1c}$ at the ambient temperature.

The current study was aimed to substantiate the utility of glucose vacutainer for HbAlc assay by addressing these lacunae in a systematic manner: i) By incorporating both diabetic and non-diabetic subjects, ii) By covering different ranges of $\mathrm{HbA} 1 \mathrm{c}$ i.e. $(<6.0 \%$, $6.0-8.0 \%, 8.1-10.0 \%, 10.1-12.0 \%$ and $12.1-16.0 \%)$; iii) By evaluating the reproducibility and repeatability of HbA1c, iv) By studying the stability of HbAlc at ambient temperature till $72 \mathrm{hrs}$ of sample collection.

\section{MATERIALS AND METHODS Recruitment of Subjects}

A total of 125 subjects, which includes 25 (15 men and 10 women) non-diabetic controls and 100 (60 men and 40 women) diabetic patients were enrolled for this study at the out-patient department of Nizam's Institute of Medical Sciences, Hyderabad, India. The mean ages of non-diabetic and diabetic subjects were $55.8 \pm 11.9 \mathrm{yrs}$ and $59.2 \pm 12.9 \mathrm{yrs}$, respectively. The Institutional Ethical Committee of Nizam's Institute of Medical Sciences, Hyderabad, India approved the study protocol. Informed consent was obtained from all the study participants.

\section{Specimen collection and Biochemical testing}

Whole blood samples were collected in two Becton Dickinson (BD) vacutainers i.e. $\mathrm{NaF} / \mathrm{Na}_{2}$ EDTA $(3 \mathrm{mg} / 6 \mathrm{mg})$ and $\mathrm{K} 2$ EDTA $(5.4 \mathrm{mg})$ from each participant. In these parallel samples, HbA1c levels were measured by ion exchange high performance liquid chromatography (HPLC) using Bio-Rad D-10 ${ }^{\mathrm{TM}}$ Dual program. The samples are automatically diluted on the D-10 and injected into the analytical cartridge. The D-10 delivers a programmed buffer gradient of increasing ionic strength to the cartridge, where the hemoglobins are separated based on their ionic interactions with the cartridge material. The separated hemoglobins then pass through the flow cell of the filter photometer, where changes in the absorbance $415 \mathrm{~nm}$ are measured. The HbAlc area is calculated using an exponentially modified Gaussian (EMG) algorithm that excludes the labile A1c and carbamylated peak area from the A1c peak area. In five representative samples stored at ambient temperature, HbA1c is repeated at 24 $\mathrm{hr}, 48 \mathrm{hr}$ and $72 \mathrm{hr}$ after collection. In two representative samples, assay was performed in 20 replicates to evaluate repeatability and reproducibility.

\section{STATISTICAL ANALYSIS}

Spearman rank correlation coefficient (r) was used to compare HbA1c levels in both vacutainers. Paired t-test was used to assess whether there is any statistically significant difference between HbA1c levels in both vacutainers. Bland and Altman analysis was performed by plotting average vs. difference plots that indicate the bias between parallel samples. Coefficient of variation (CV\%) was used to calculate the precision in HbA1c measurement. Paired t-test was used to study stability of $\mathrm{HbA} 1 \mathrm{c}$ at ambient temperature up to $72 \mathrm{hrs}$ of blood collection.

\section{RESULTS \\ Comparison of $\mathrm{HbA1C}$ in $\mathrm{NaF} / \mathrm{Na}_{2}$ EDTA and $\mathrm{K} 2$ EDTA}

In non-diabetic subjects, we observed HbAlc levels in the range of $4.8-6.0 \%$. The HbA1c levels in this group showed a correlation coefficient of 0.96 . In all the diabetic subgroups, the correlation coefficient value is consistent i.e. 0.99. Cumulatively, across the broad range of $4.8-16.0 \% \mathrm{HbA1c}$ the correlation was 0.99 (Figure-1). The regression equation showed a slope value of 0.998 and intercept value of -0.023 .

Paired t-test showed that mean difference between both methods is 0.041 (95\% CI: 0.021-0.061). The HbA1c levels in K2 EDTA and $\mathrm{NaF} / \mathrm{Na}_{2}$ EDTA were $9.08 \pm 2.92 \%$ vs. $9.04 \pm 2.92 \% \quad(\mathrm{p}<0.0001)$, respectively.

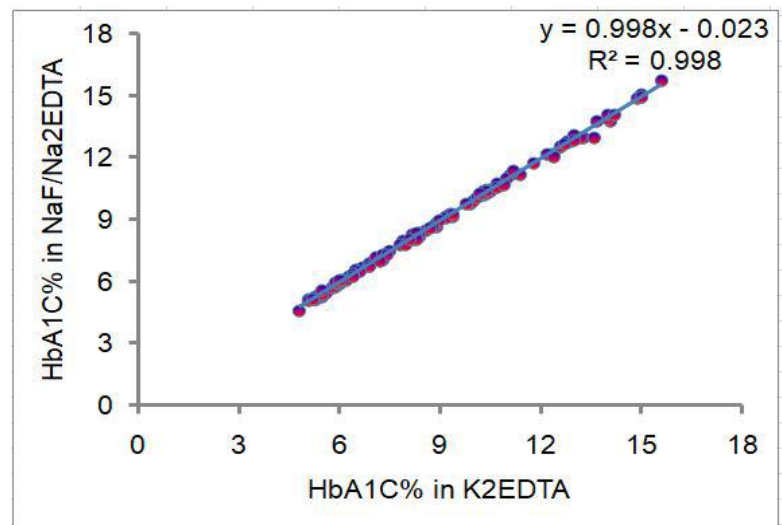

Fig-1: Correlation between $\mathrm{NaF} / \mathrm{Na}_{2}$ EDTA and K2 EDTA anticoagulants 
This illustrates correlation plot of $\mathrm{HbAlc}$ levels in $\mathrm{NaF} / \mathrm{Na}_{2}$ EDTA and $\mathrm{K} 2$ EDTA. The correlation coefficient is 0.99 . The regression equation depicted a slope value of 0.998 and intercept value of 0.023 .

\section{Bland and Altman analysis}

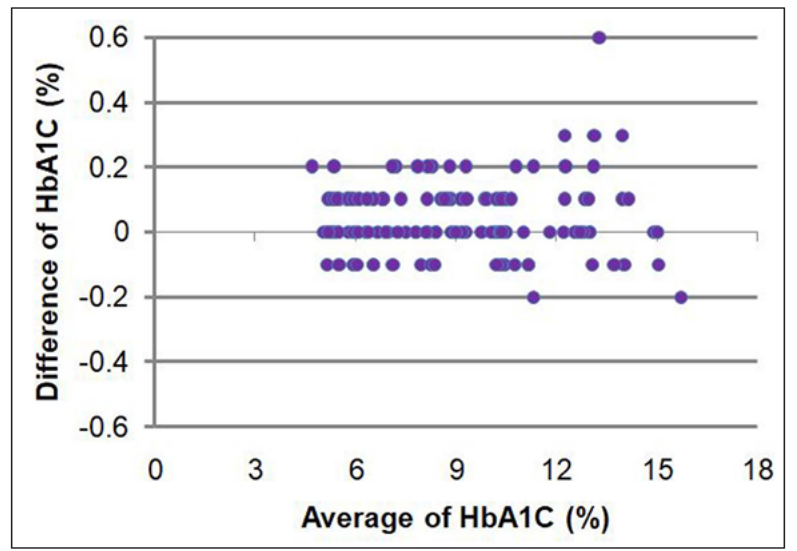

Fig-2: Bland and Altman analysis of HbA1c levels in $\mathrm{NaF} / \mathrm{Na} 2$ EDTA and K2 EDTA anticoagulants

Bland and Altman analysis was performed by plotting average vs. difference between HbA1c levels in $\mathrm{NaF} / \mathrm{Na}_{2}$ EDTA and K2 EDTA. The bias between the methods was \pm 0.02 .

As shown in Figure-2, average vs. difference plot was computed to assess the bias in measuring HbA1c levels in both the anticoagulants. The bias between methods is -0.22 to $+0.22 \%$.

\section{Stability of HbA1c at ambient temperature}
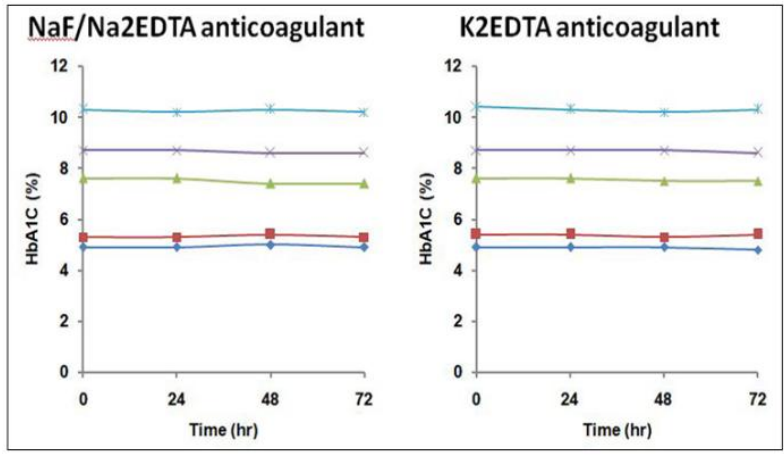

Fig-3: Stability of HbA1c at ambient temperature $\mathrm{NaF} / \mathrm{Na}_{2}$ EDTA and K2 EDTA anticoagulants

In five representative subjects, parallel samples of $\mathrm{NaF} / \mathrm{Na}_{2}$ EDTA and K2 EDTA were used to measure $\mathrm{HbA} 1 \mathrm{c}$ at different time intervals till $72 \mathrm{hrs}$ of sample collection. No significant difference was obtained from the time of collection to till $72 \mathrm{hrs}$.

As shown in Figure 3, five representative samples whose $\mathrm{HbA1c}$ levels are in the range 4.9$10.4 \%$ were tested for stability at room temperature at $24 \mathrm{hr}, 48 \mathrm{hr}$ and $72 \mathrm{hr}$ after sample collection. In K2 EDTA, the CV ranged from $0.58-1.02 \%$ while in $\mathrm{Na}_{2}$ EDTA the CV ranged from $0.55-1.53 \%$.

\section{Repeatability and reproducibility}

Two representative samples were processed 20 times to check their repeatability and reproducibility. The coefficient of variation was $0.71 \%$ and $1.56 \%$ in these two samples. The measurement of uncertainty was 0.076 and 0.16 in these samples. Levy Jenning plot of the mean of both samples in 20 different runs showed values within 1 SD (Figure-4).

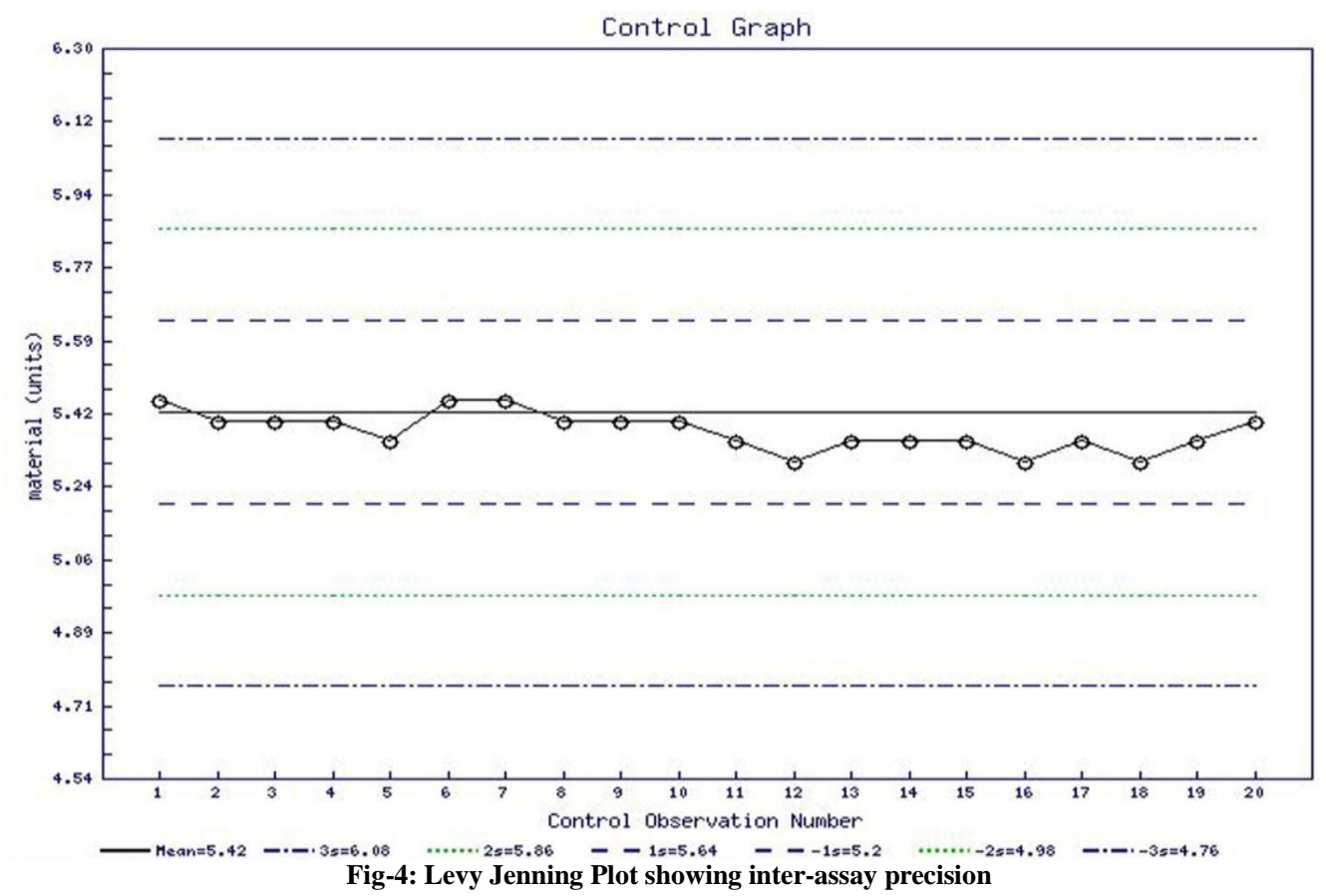


The mean values of two representative samples processed in 20 runs were depicted in the Levy Jenning plot. The values are within 1SD.

\section{DISCUSSION}

This is the first systematic investigation in the clinical utility of glucose vacutainer for HbA1c measurement. This study substantiates that same vacutainer can be used for glucose and $\mathrm{HbA1c}$ measurements with high precision across a broad range of HbA1c (4.8-16\%). The HbA1c levels were stable till $72 \mathrm{hr}$ of collection at ambient temperature. Although, mean difference between both the methods i.e. 0.04 is statistically significant, it will not have practical implications on its clinical utility as the difference is small. The slope value of 0.998 and intercept value of 0.023 in the regression equation substantiate this statement further. This is consistent with findings of Chakraborty et al., who demonstrated slope value of 1.00 and intercept value of 0.14 in the regression model of $\mathrm{HbA} 1 \mathrm{c}$ in EDTA vs. Fluoride vacutainers [9]. In addition to the three studies mentioned earlier, two more studies compared HbA1c levels in sodium fluoride and K2 EDTA in limited number of subjects, which are in agreement with our findings $[10,11]$.

Vrtaric A and his colleagues have observed that there is no clinically and statistically significant bias between K2-EDTA and K3-EDTA HbA1c measurements [12]. Also, Sarmah D et al., have demonstrated that $\mathrm{HbAlc}$ values in fresh and stored whole blood sample does not change when analysed in K3-EDTA, Na-citrate, lithium-heparin and $\mathrm{Na}$ fluoride/ $\mathrm{Na}_{2}$ EDTA anticoagulant vials [13]. Recently, Kumawat $\mathrm{R}$ and her colleagues have also observed that there was no significant difference in the HbA1c values in samples collected in sodium fluoride/potassium oxalate and EDTA tubes at the time of initial estimation and after 7 days of collection under proper storage [14].

Our study demonstrated that there was no significant difference in the HbA1c values when it was measured in $\mathrm{NaF} / \mathrm{Na}_{2}$ EDTA. In view of increasing incidence of diabetes mellitus in India, there is an increase in the numbers of $\mathrm{HbA} 1 \mathrm{c}$ test requests from the treating physicians. It is known that $\mathrm{HbAlc}$ test is costlier than blood glucose test; use of a separate K2 EDTA vacutainer for $\mathrm{HbA1c}$ testing increases the cost further. If both plasma glucose and $\mathrm{HbAlc}$ can be performed from the same $\mathrm{NaF} / \mathrm{Na}_{2}$ EDTA vacutainer, there is no need of additional blood sample which in turn reduces the cost associated with $\mathrm{HbA1c}$ testing.

The major strengths of the current study are: i) Inclusion of non-diabetic and diabetic subjects, ii) Equal representation of subjects across HbA1c measurable range of $4.8-16 \%$, iii) Comparison of HbA1c levels between both vacutainers using different statistical approaches such as correlation coefficient, regression equation, paired t-test, etc, iv) Demonstration of repeatability and reproducibility in $\mathrm{HbA1c}$, and v) Stability assessment at ambient temperature up to 72 hrs of blood sample collection.

\section{CONCLUSION}

The current study along with the previous pilot studies emphasized that single BD glucose vacutainer is sufficient and can be safely used for $\mathrm{HbAlc}$ measurement from whole blood and glucose measurement from plasma after centrifugation of same sample, which is cost-effective in terms of laboratory perspective, particularly medium and large-sized laboratories, and facilitates timely therapeutic intervention in terms of clinical perspective. Future studies are warranted to examine the utility of glucose vacutainers in non-HPLC based HbA1c assays.

\section{REFERENCES}

1. Bunn, H. F., Haney, D. N., Gabbay, K. H., \& Gallop, P. M. (1975). Further identification of the nature and linkage of the carbohydrate in hemoglobin A1c. Biochemical and biophysical research communications, 67(1), 103-109.

2. Koenig, R. J., Peterson, C. M., Jones, R. L., Saudek, C., Lehrman, M., \& Cerami, A. (1976). Correlation of glucose regulation and hemoglobin AIc in diabetes mellitus. New England Journal of Medicine, 295(8), 417-420.

3. Unnikrishnan, R., Anjana, R. M., \& Mohan, V. (2016). Diabetes mellitus and its complications in India. Nature Reviews Endocrinology, 12(6), 357370.

4. Das, A. K., Saxena, G., \& Naik, S. (2019). HbA1C in Management of Type II Diabetes Mellitus: A Cross-sectional Survey of Indian Physicians. Journal of The Association of Physicians of India, 67 (7):18-21.

5. Pettus, J. H., Zhou, F. L., Shepherd, L., Preblick, R., Hunt, P. R., Paranjape, S., ... \& Edelman, S. V. (2019). Incidences of severe hypoglycemia and diabetic ketoacidosis and prevalence of microvascular complications stratified by age and glycemic control in US adult patients with type 1 diabetes: a real-world study. Diabetes care, 42(12), 2220-2227.

6. Mohan, I. K., Sreedevi, N. N., Baba, K. S. S., Bhaskar, M. V., Hussain, T., Alrokayan, S. A., \& Naushad, S. M. (2019). Application of multiple linear regression and machine learning algorithms to elucidate the association of poor glycemic control and hyperhomocysteinemia with microalbuminuria.Indian Journal Biochem Biophys, 56(2):150-154.

7. Sharma, B., Sarmah, D., \& Sonker, P. (2013). Effect of Different Anticoagulants on HbA1c Estimation and its Stability. Journal Lab Physicians, 5(2):143-144.

8. Fournier, J. E., Northrup, V., Canales, D. D., Moore, C., \& Shea, J. L. (2018). The Effects of Storage and Additives on Postmortem HbA1c 
Measurements. Journal Forensic Science, 63(6):1870-1874.

9. Chakraborty, S., Ghosh, S.S., Das, A., Sawant, P., \& Kallner, A. (2015). Can EDTA, EDTA-fluoride, and buffered citrate tubes be used for measurement of HbA1c on the Bio-Rad D10? Clinical Chemistry Lab Med, 53(1):e5-8.

10. Mailankot, M., Thomas, T., Praveena, P., Jacob, J., Benjamin, J. R., \& Vasudevan, D. M. (2012). Various Anticoagulants and Fluoride do not Affect HbA1C Level. Indian Journal Clinical Biochemistry, 27(2), 209.

11. Singh, B., Singh, K., \& Mahdi, A. A. (2014). Estimation of glycated hemoglobin sample in sodium fluoride vacutainer: A better option. Journal of Clinical \& Experimental
Investigations/Klinik ve Deneysel Arastirmalar Dergisi, 5(2):336-338.

12. Vrtaric, A., Filipi, P., Hemar, M., Nikolac, N., \& Simundic, A. M. (2016). K2-EDTA and K3EDTA Greiner Tubes for $\mathrm{HbA1c}$ Measurement. Laboratory medicine, 47(1), 39-42.

13. Sarmah, D., \& Sharma, B. (2013). A comparative evaluation of $\mathrm{HbA1c}$ measurement in different anticoagulant vials and its stability on storage. International Journal of Current Research and Review, 5(11), 73.

14. Kumawat, R., Patel, S., Saini, V., \& Kaur, C. (2019). Can Oxalate-Fluoride Vacutainers Be Used for Measurement of CoRequested HbA1c along with Blood Glucose? - A Hospital Based Study in Northern India. International Journal of Research and Review, 6(6):287-93. 